\title{
Analysis and Implementation of a Low-cost High Frequency Inverter for Driving a Nonlinear Load
}

\author{
Gang-Youl Jeong \\ Department of Electronic Information Engineering, Soonchunhyang University \\ gangyoul@sch.ac.kr
}

\begin{abstract}
This paper presents the analysis and implementation of a low-cost high frequency inverter for driving a nonlinear load. The proposed high frequency inverter utilizes the structure of a switching power amplifier circuit that is very simple and highly efficient. The proposed inverter can drive a nonlinear load such as a cold-cathode fluorescent lamp. The drive circuit of the proposed inverter is operated by a clock generator circuit and a comparator. In this paper, the circuit of the proposed inverter is introduced, the operational principle is analyzed, and a prototype implementation is discussed. Experimental results using a cold-cathode fluorescent lamp demonstrate the performance and characteristics of the proposed inverter for driving a nonlinear load.
\end{abstract}

Keywords: High frequency, Inverter, Nonlinear load, Boost mode, Resonance mode

\section{Introduction}

There are many nonlinear loads in modern industry $[1,2]$, for example, the cold-cathode fluorescent lamp (CCFL). The CCFL has a nonlinear load characteristic, as shown in Figure 1. The best method of driving a load with a nonlinear characteristic such as the CCFL is to use a power source of the current source type. When the CCFL is in a compliance (open-circuit) state, the CCFL voltage can rise to the avalanche onset level. Then, as soon as ionization occurs, the CCFL voltage will drop and the desired operating point will be reached immediately. In such applications, a single frequency component sinusoidal drive is preferred to maximize efficiency and minimize electromagnetic interference (EMI) [3].

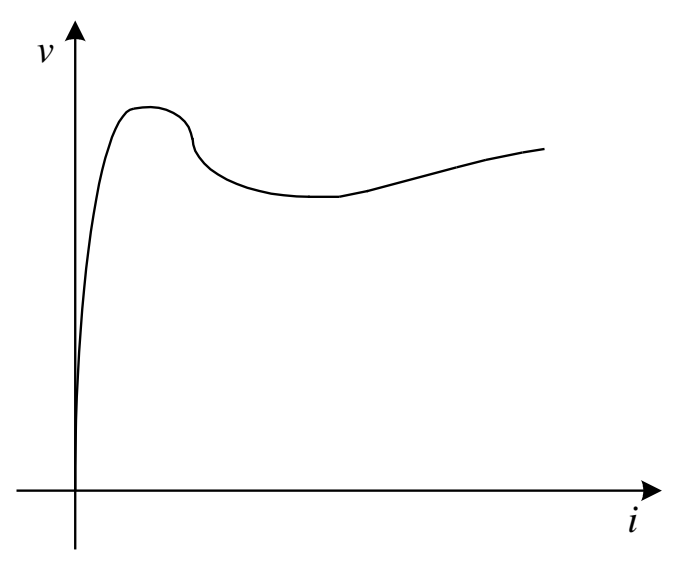

Figure 1. Nonlinear Load Characteristic 
The high frequency inverter for driving a nonlinear CCFL load is powered by an 8-20 V DC battery supply or an AC-DC adaptor equivalent. The inverter is an electronic device that converts input DC power to an AC power. The CCFL requires 1-2 $\mathrm{kV}$ to initiate, and its circuit efficiency and size are critical. The CCFL typically operates with sinusoidal voltage of $700-800 \mathrm{~V} \mathrm{AC}$ and a current of 7-10 mA rms. The operation frequency is normally recommended between $25-85 \mathrm{kHz}$, and a sinusoidal lamp voltage waveform is preferable. Additionally, a dimming control capability is another desirable feature for such applications. These formidable requirements demand a highly efficient conversion topology and maximum circuit integration [4-7].

The most frequently used inverter circuit for nonlinear loads has a push-pull current-source parallel-resonant inverter (CSPRI) with a buck converter added for power regulation. In CSPRI circuits, the output voltage is sinusoidal, and the timing is usually based on the resonance of the transformer's magnetizing inductance and a capacitance in parallel with the primary winding [8]. However, the CSPRI/buck-pre-regulator combination has several disadvantages such as low efficiency due to two power switches in series, reduced efficiency due to distorted lamp voltage and current, large EMI due to hard-switching of the buck preregulator and unrelated switching frequencies of the pre-regulator and the inverter, and high cost due to complex circuit topology and an expensive control IC.

The current synchronous zero voltage switching (CS-ZVS) half-bridge inverter [9] is more efficient than the CSPRI/buck pre-regulator combination, but it also has a rather complex topology, with two controlled switches, two magnetic elements, and three capacitors in the power train. Other drawbacks of the circuit are that the leakage inductance of the transformer is not absorbed in the load network, leading to significant CCFL voltage distortion and reduced efficiency, the topology is claimed to be proprietary and can be used only with an expensive single-source dedicated control IC, and when using a dedicated control IC, the inverter requires either a complementary metal-oxide semiconductor field effect transistors (MOSFETs), or alternatively it would require a floating driver of the MOSFETs.

The high frequency inverter for driving a nonlinear load is based on the conventional switching power amplifier circuit topology [10-13]. The circuit has many advantages compared with the CSPRI/buck-pre-regulator combination and CS-ZVS half-bridge inverter. The advantages are that only one switch is required; the leakage inductance of the transformer is absorbed in the load network; the inverter is fed with an inductance, and therefore, the interference current reflected back to the source is small; the switch voltage waveform is smooth with a low $d v / d t$, and therefore, the capacitive interference is reduced; and the topology is public domain information.

In this paper, a low-cost high frequency inverter for driving a nonlinear load is analyzed and implemented. Because the proposed inverter uses simple drive and control circuits, its total structure can be simplified while presenting high efficiency characteristics. The proposed inverter is capable of powering a nonlinear load, such as a CCFL. The drive circuit of the proposed inverter utilizes a clock generator and a comparator. In this paper, the circuit of the proposed inverter is introduced, its operating principle is described and analyzed, and a brief prototype implementation is presented. It is shown through experimental results that the proposed inverter performs well when driving a nonlinear load - specially a CCFL.

\section{Operation Principle and Analysis}

The resonant inductor in the conventional switching power amplifier is in series with the primary winding of the transformer. This allows the absorption of the leakage inductance of the transformer in the resonant network, which leads to a reduction in overall cost. The new switching power amplifier of the proposed inverter is based on this design. 
As shown in Figure 2(a), the topology used in the proposed inverter modifies the conventional switching power amplifier circuit. It consists of a switch $S$ with a built-in antiparallel diode $D_{r}$, a resonant capacitor $C_{r}$, and a transformer $T$ that feeds the load $R_{L}$, where $R_{L}$ denotes an equivalent resistor of the CCFL. To make the inverter as simple as possible, the transformer and inductor are considered as one element, with the combined magnetic device viewed as two loosely coupled inductors wound a single core. This integrated magnetic structure can be represented by the analytic model shown in figure 2(b). In this model, the transformer $T$ in figure 2(a) comprises the ideal transformer $T$, the magnetizing inductance $L_{m}$, and the primary and secondary leakage inductance $L_{l \mathrm{k} 1}$ and $L_{l k 2}$, respectively.

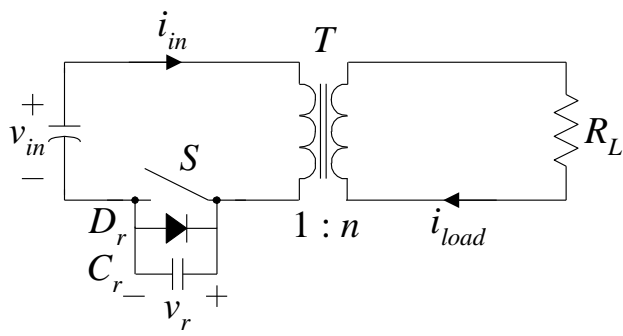

(a) Real circuit of the proposed switching power supply

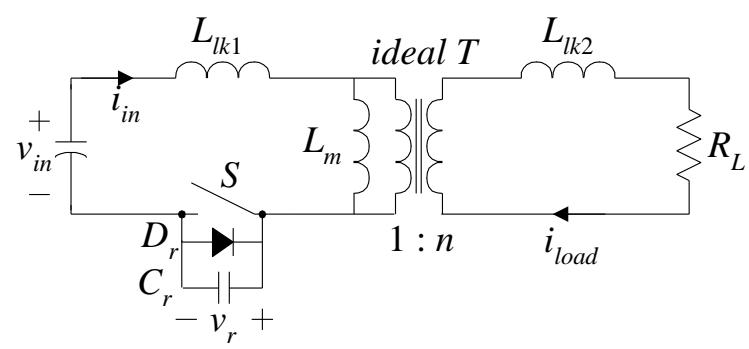

(b) Analytic model of the switching power supply with integrated magnetic structure

Figure 2. Circuit Topology Used in the proposed Switching Power Supply

The basic operation of the proposed inverter is analyzed by considering the simplified equivalent model of Figure 3, where the secondary leakage inductance $L_{l k 2}$ and load $R_{L}$ are reflected to the primary, and the magnetizing inductance $L_{m}$ is replaced by a current source $I_{m}$. The latter substitution is justified under the assumption that the impedance of $L_{m}$ is much larger than the total reflected impedance of the secondary.

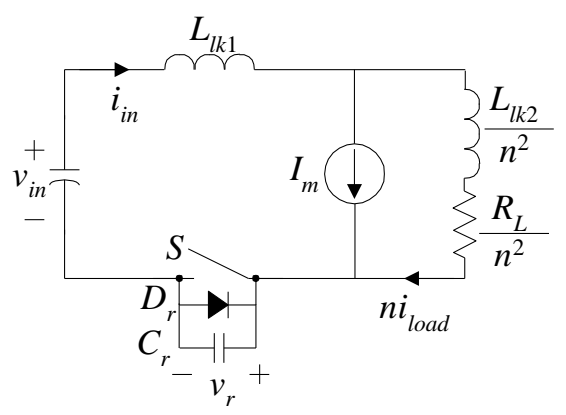

Figure 3. Simplified Model of the Inverter for Analysis 
As shown in Figure 4, the inverter has two operational modes: the boost mode and the resonance mode. Figure 5 shows its theoretical operational waveform.

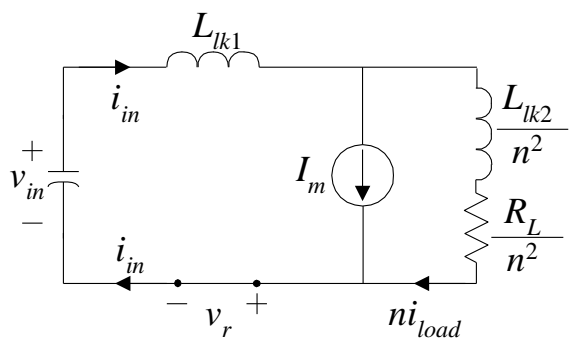

(a) Boost mode

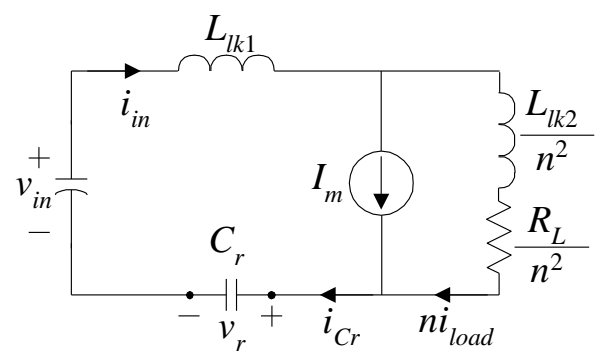

(b) Resonance mode

\section{Figure 4. Operational Modes of the Inverter}

During the boost mode in Figure 4(a), either the switch or the diode is conducting. In this time interval, the capacitor is shorted to ground and the reflected load current $n i_{\text {load }}$ at the reflected branch increases exponentially. During the resonance mode in figure 4(b), the switch is turned off, and the inductive branch and the capacitor form a resonant network, which forces the voltage $v_{r}$ across the switch to follow a sinusoidal waveform. In a properly designed inverter, the sinusoidal waveform eventually returns to the zero level after the antiparallel diode catches. The gate-source voltage $v_{g s}$ can then be applied to turn on the switch under the ZVS condition. The resonant capacitor $C_{r}$ reduces the $d v / d t$ across the switch at turn-off, which reduces switching losses and allows high frequency operation.

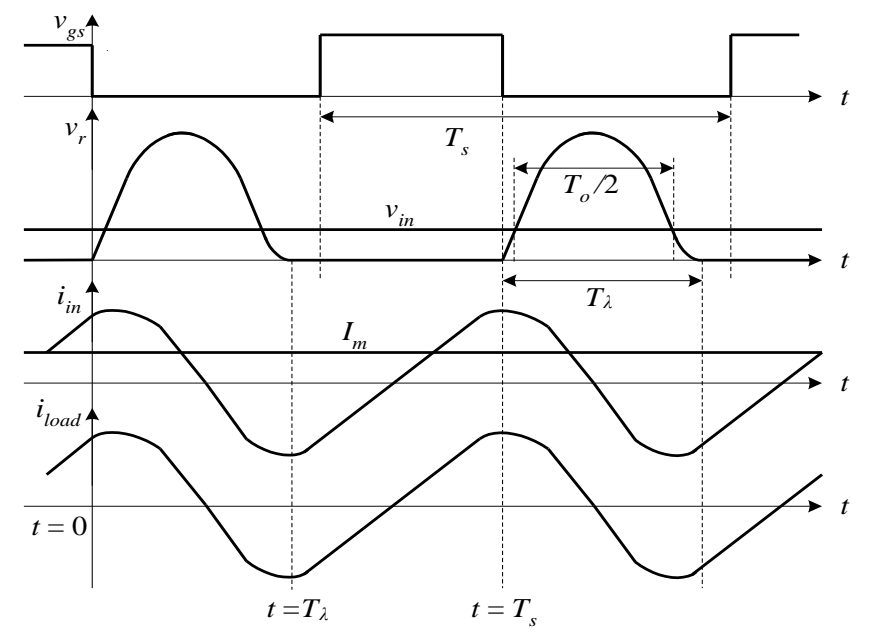

Figure 5. Theoretical Operational Waveform of the Inverter 
The theoretical analysis of the inverter is developed under the assumptions that the switch, diode, capacitor, and inductor are ideal, the magnetizing inductance $L_{m}$ is much larger than the leakage inductances $L_{l k 1}$ and $L_{l k 2}$, and the parasitic inductances and capacitances are negligibly small.

The switch voltage $v_{r}$ during the resonant period $T_{\lambda}$ can be derived from the following set of differential equations:

$$
\begin{aligned}
I_{C r} & =I_{m}+n i_{\text {load }} \\
v_{r} & =\frac{1}{C_{r}} \int i_{C r} d t \\
V_{\text {in }} & =L_{l k 1} \frac{d i_{C r}}{d t}+\left(\frac{L_{l k 2}}{n^{2}} \frac{d}{d t}+R_{r}\right) n i_{\text {load }}+v_{r}
\end{aligned}
$$

where $L_{l k 1}$ is the primary inductance, $L_{l k 2} / n^{2}$ is the reflected secondary leakage inductance, $C_{r}$ is the resonant capacitance, $n$ is the turn ratio, $R_{r}=R_{L} / n^{2}$ is the reflected load resistor, $R_{L}$ is the lamp resistance, $n i_{\text {load }}$ is the reflected load current, and $I_{m}$ is the amplitude of the current source. The general solution of the set of differential equations (1) has the following form:

$$
v_{r}=\left[A \sin \omega_{r} t+B \cos \omega_{r} t\right]^{-\frac{R_{r}}{2 L_{r}} t}+V_{\text {in }}+R_{r} I_{m}
$$

where $L_{r}=L_{l k 1}+L_{l k 2} / n^{2}, \omega_{r}=\omega_{0} \sqrt{1-(1 / 2 Q)^{2}}, Q=\left(\sqrt{L_{r} / C_{r}}\right) / R_{r}$ and $\omega_{0}=1 / \sqrt{L_{r} C_{r}}$.

The integration constants $\mathrm{A}$ and $\mathrm{B}$ are found by the boundary conditions that $v_{r}=0$ at time $t=0$ and time $t=T_{\lambda}$. Using these conditions, the switch voltage $v_{r}$ is obtained as

$$
v_{r}=\left(V_{i n}+R_{r} I_{m}\right)\left[\left(\frac{\cos \theta_{\lambda}-e^{\alpha}}{\sin \theta_{\lambda}}\right) \frac{\sin \left(\theta_{\lambda} x\right)}{e^{\alpha x}}-\frac{\cos \left(\theta_{\lambda} x\right)}{e^{\alpha x}}+1\right]
$$

where $\alpha=\theta_{r} R_{n} / \sqrt{4-R_{n}{ }^{2}}, \theta_{\lambda}=\omega_{r} T_{\lambda}, x=t / T_{\lambda}$, and the normalized load resistance $R_{n}=1 / Q$.

In steady state, the average voltage of $v_{r}$ is nearly equal to $V_{\text {in }}$ as follows:

$$
V_{\text {in }}=\frac{1}{T_{s}} \int_{0}^{T_{s}} v_{r} d t
$$

Using $v_{r}=0$ during the $T_{\lambda}<t<T_{s}$ period,

$$
V_{\text {in }}=\frac{\alpha}{\pi R_{n}} \frac{f_{s}}{f_{0}} \int_{0}^{1} v_{r} d t
$$

where $f_{o}=\omega_{o} / 2 \pi$ is the resonant frequency and $f_{s}$ is the switching frequency.

From (5), assuming that the inverter is lossless and equating input power to output power, the normalized load current $I_{\text {load }, n}=n I_{\text {load }, r m s} /\left(V_{i n} / Z_{r}\right)$ is given by

$$
I_{\text {load }, n}=\frac{1}{R_{n}} \sqrt{\frac{\pi\left(f_{0} / f_{s}\right)}{\frac{\theta_{\lambda} R_{n}}{4 \alpha \sin \theta_{\lambda}}\left(2 \cos \theta_{\lambda}-e^{\alpha}-e^{-\alpha}\right)+\frac{\alpha}{R_{n}}}-1}
$$

where $I_{\text {load,rms }}$ is the rms load current, and $Z_{r}=\sqrt{L_{r} / C_{r}}$ is the characteristic impedance of the $L_{r}-C_{r}$ resonant circuit. The theoretical results clearly indicate that the inverter operates as a current source and that the output current magnitude is controlled by the switching frequency $f_{s}$.

\section{Prototype Implementation}

In order to show that the proposed high frequency inverter operates effectively as a high frequency inverter for driving a nonlinear load, a prototype inverter is implemented as shown in the control block diagram in Figure 6. The proposed high frequency inverter is very simple. 
The total system of the proposed inverter consists of two parts: a controller part and a power circuit part. The controller part includes a protector circuit and consists of a pulse generator for pulse-width modulation (PWM), a comparator for comparison of the real output and reference value and the reference waveform generator for the dimming control of the nonlinear load. The power circuit part is composed of one MOSFET, an input capacitor and a main transformer.

In order to reduce the total size of the proposed inverter, the transformer size is reduced, and the proposed inverter is operated at high frequency, $f_{s}=50 \mathrm{kHz}$. The magnetizing inductance of the transformer primary seen from the transformer secondary is approximately $L_{m}=200 \mathrm{mH}$, and the DC input voltage is $V_{i n}=12 \mathrm{~V}$. The nonlinear load utilized in this experiment is a CCFL with an operating voltage and current of $800 \mathrm{~V}$ and $10 \mathrm{~mA} \mathrm{AC} \mathrm{rms,}$ respectively, with an operating frequency of approximately $50 \mathrm{kHz}$.

For the dimming control of the lamp, two PWM pulses are modulated - the switching frequency $50 \mathrm{kHz}$ and the dimming frequency $100 \mathrm{~Hz}$. The dimming control is performed by regulation of the lamp current based on the on-duty period of dimming frequency. The lamp brightness reference is given from a $5 \mathrm{~V}$ variable resistor.

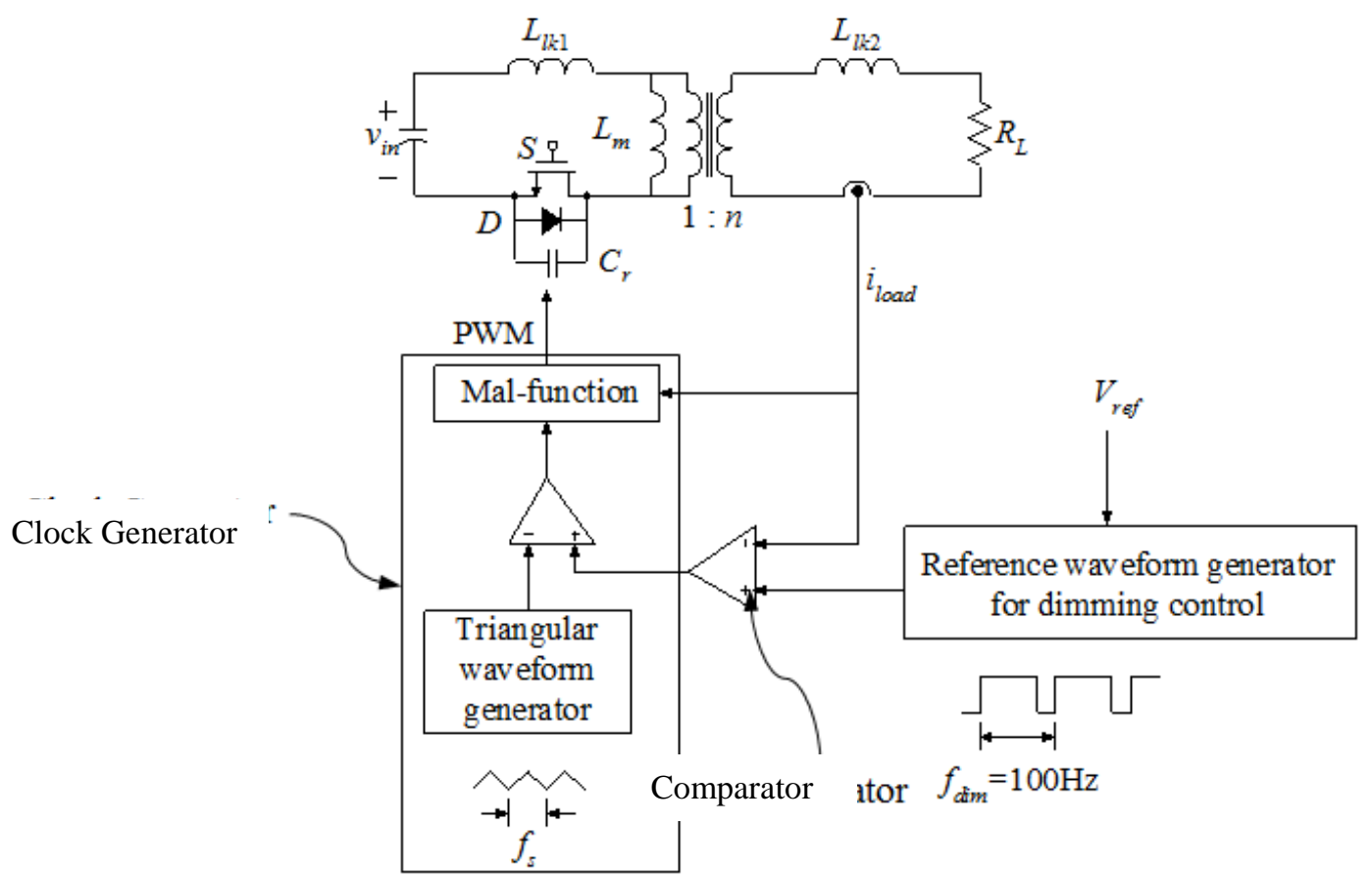

Figure 6. Total Control Block Diagram of the Propo Sed Inverter

\section{Experimental Results}

In order to verify the validity of the proposed inverter, a prototype inverter was built according to the design described in Section 3. From this prototype, experimental results were obtained, as described below.

Figure 7 shows experimental waveforms of the gate drive $v_{g s}$ and the switch voltage $v_{r}$. These waveforms show that ZVS of the proposed inverter is achieved and the inverter satisfactorily generates PWM pulses and demonstrates stable operation. 

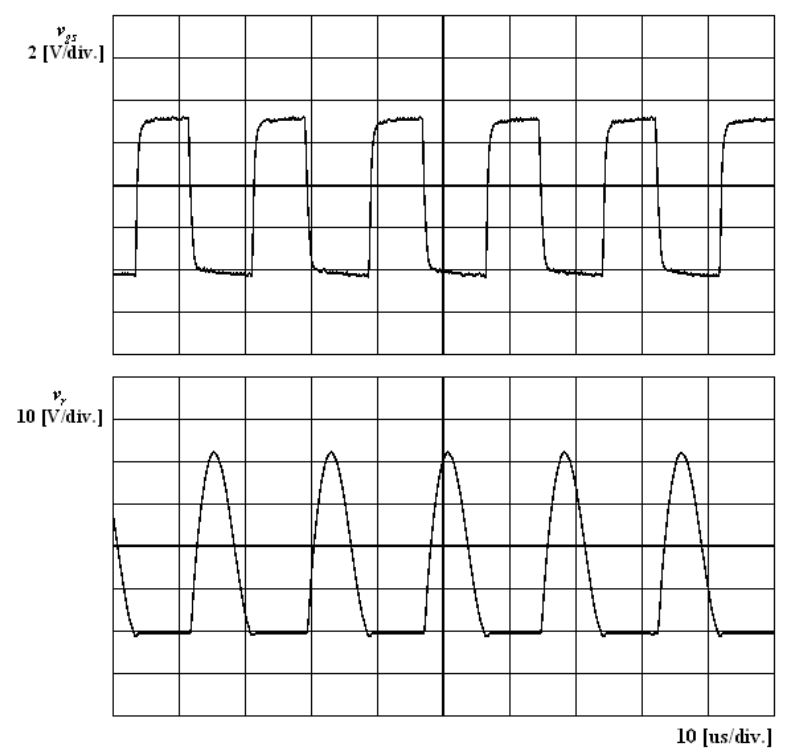

Figure 7. Experimental Waveforms of the Gate Drive Voltage $v_{g s}$ and the Switch Voltage $v_{r}$

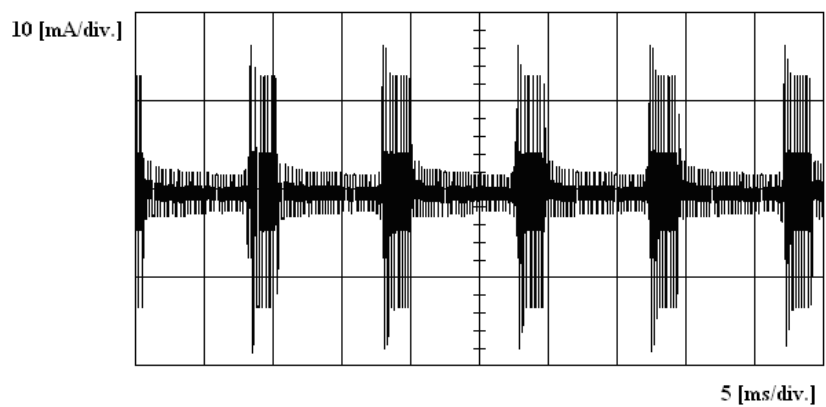

(a) $25-\%$ dimming

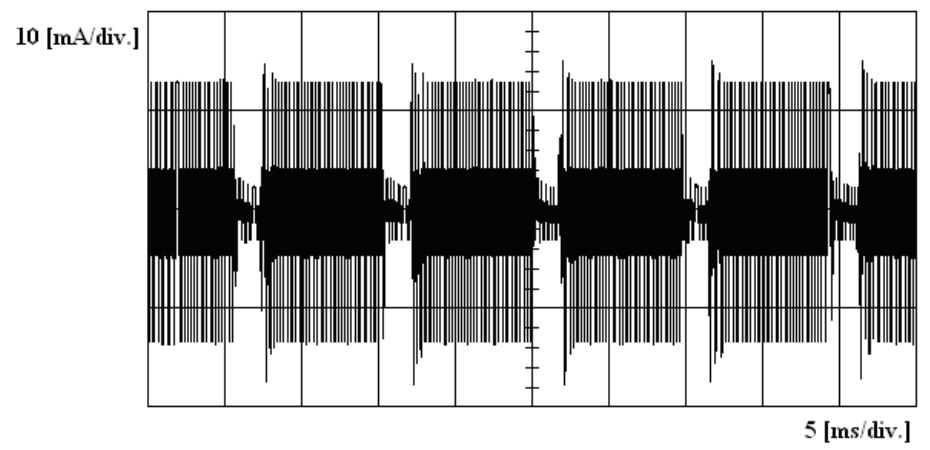

(b) $80-\%$ dimming

Figure 8. Lamp Current Waveforms at Each Dimming State 

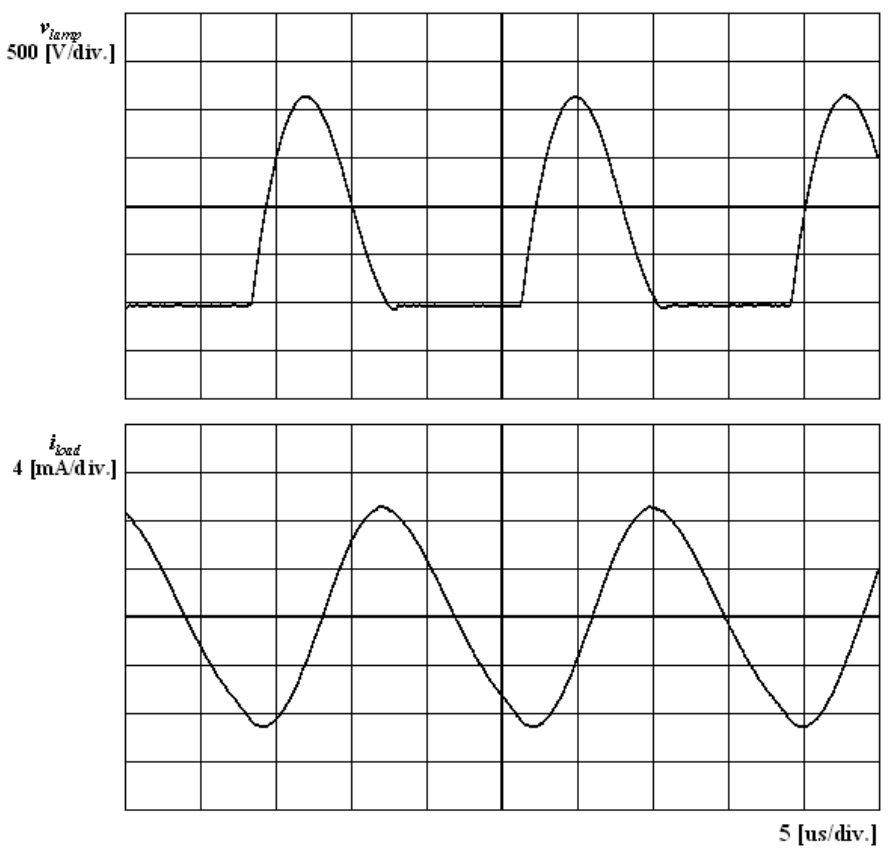

Figure 9. Experimental Waveforms of the Lamp Voltage $v_{\text {lamp }}$ and the Lamp Current $i_{\text {load }}$

Figure 8 shows experimental waveforms of the lamp current waveforms at $25-\%$ dimming (a) and 80-\% dimming (b), respectively, where the dimming corresponds to a change in the lamp's brightness. It can be seen that the initial lamp turn-on current is nearly half that of a conventional inverter.

Figure 9 shows experimental waveforms of the voltage $v_{\text {lamp }}$ across the lamp and the lamp current $i_{\text {load }}$. These waveforms also illustrate good, stable performance of the system.

\section{Conclusion}

In this paper, the design of a low-cost high frequency inverter for driving a nonlinear load has been described, analyzed, and implemented. The proposed inverter uses simple drive and control circuits; thus while its overall structure is simple, it exhibits highly efficient characteristics. The proposed inverter can power a nonlinear load, such as a CCFL. The drive circuit of the proposed switching power supply utilizes a clock generator and a comparator. In this paper, the circuit of the proposed is introduced, and its operational principle described and analyzed and the implementation of a prototype is also demonstrated. It is shown through experimental results using a CCFL that the proposed inverter exhibits good performance when driving a nonlinear load.

\section{ACKNOWLEDGEMENTS}

This work was supported by the Soonchunhyang University Research Fund.

\section{References}

[1]. M. G. Kim, G. Y. Park, D. H. Yoo and G. Y. Jeong, Int. Jour. of contr. and Auto, vol. 7, no. 4, (2014).

[2]. S. H. Kwon, D. H. Yoo and G. Y. Joeng, Int. Jour. of contr. and Auto, vol. 7, no. 6, (2014).

[3]. J. A. Donahue and M. M. Jovanovic, APEC, (1994), Orlando, USA. 
[4]. G. H. Kweon, Y. C. Lim and S. H. Yang, ISIE, (2001), Pusan, Korea.

[5]. S. W. Lee, D. Y. Ko, D. Y. Huh and Y. I. Yoo, APEC, (2001), Anaheim, USA.

[6]. M. S. Lin, W. J. Ho, F. Y. Shih, D. Y. Chen and Y. P. Wu, IEEE Trans. on Ind. elec., vol. 45, no. 2, (1998).

[7]. R. Redl and K. Arakawa, APEC, (1997), Atlanta, USA.

[8]. R. L. Bright, G. F. Pittman and G. H. Royer, Electrical Manufacturing, vol. 54, no. 1, (1954).

[9]. M. K. Nalbant, APEC, (1995), Dallas, USA.

[10].N. O. Sokal and A. D. Sokal, IEEE J. Solid-State Circuits, SC, vol. 10, (1975).

[11].R. Redl and N. O. Sokal, HFPC, (1989), San Jose, USA.

[12].G. Y. Jeong, Journal of the Korea Academia-Industrial Cooperation Society, vol. 11, no. 2, (2010).

[13].M. Chakraverty, S. Mandava and G. Mishra, Int. Jour. of contr. And Auto., vol. 3, no. 2, (2010).

\section{Authors}

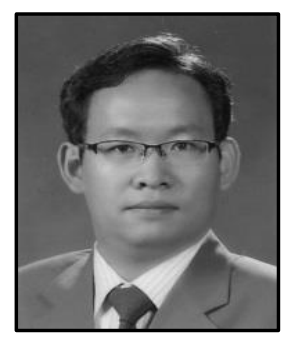

Gang-Youl Jeong received his M.S. and Ph.D. degrees in Electronic and Electrical Engineering from POSTECH (Pohang University of Science and Technology), Korea, in 1999 and 2002, respectively. He has been an associate professor in Department of Electronic Information Engineering, Soonchunhyang University, Korea. His research interests include DC-DC power converter, AC-DC high frequency inverter, and power conversion for the renewable energy. 
International Journal of Control and Automation Vol. 8, No. 2 (2015) 